\title{
HYBRID EULERIAN-LAGRANGIAN APPROACH FOR DENSE SPRAY SIMULATIONS
}

\section{Hosein Heidarifatasmi ${ }^{1, *}$, Thorsten Zirwes ${ }^{2,1}$, Feichi Zhang ${ }^{1}$, Peter Habisreuther ${ }^{1}$ and Dimosthenis Trimis ${ }^{1}$}

${ }^{1}$ Engler-Bunte-Institute, Division of Combustion Technology, Karlsruhe Institute of Technology Engler-Bunte-Ring 1, Karlsruhe 76131, Germany hosein.heidarifatasmi@kit.edu; thorsten.zirwes@kit.edu; feichi.zhang@kit.edu; peter.habisreuther@kit.edu; dimosthenis.trimis@kit.edu; vbt.ebi.kit.edu

${ }^{2}$ Steinbuch Centre for Computing, Karlsruhe Institute of Technology Hermann-von-Helmholtz-Platz 1, 76344 Eggenstein-Leopoldshafen, Germany thorsten.zirwes@kit.edu; www.scc.kit.edu

Key words: Hybrid Eulerian-Lagrangian, Dense sprays, VOF method, OpenFOAM

\begin{abstract}
.
In this work, a hybrid Euler-Lagrangian solver for dense spray systems is developed specifically for cases where film creation by accumulation of liquid droplets at the walls plays a crucial role. EulerLagrangian solvers are commonly used to describe the spray with predefined spray characteristics. The Lagrangian particles represent liquid drops moving along the continuous gaseous phase. This approach assumes a small particle size compared to the cell size and it is unable to capture the breakup behavior of liquid jets in the presence of instabilities. VOF methods, on the other hand, are not a computationally feasible option when it comes to small droplet sizes as a result of liquid atomization because they have to be fully resolved by the computational mesh. Hence, multiscale simulations are required to bridge the gap between the two methods combining subgrid droplets in Lagrangian approaches and large liquid structures in VOF methods.

In the present work, a multiscale approach is developed where Lagrangian particles representing small droplets are tracked through the continuous phase until they hit a wall or a liquid-gas interface represented by a continuous VOF field. At the time of impact, the Lagrangian particles are removed and the mass and momentum of these particles are transferred to the VOF field. This allows having a spray consisting of subgrid droplets computed with a Lagrangian particle tracking (LPT) approach and liquid films at the walls with VOF method. The method represents a one-way coupling, converting Lagrangian particles to Eulerian liquid phase (VOF) and has been implemented into the open-source CFD code OpenFOAM. Subsequently, the solver has been tested in different scenarios to ensure mass and momentum conservation. Positive test results encouraged its use to gain insight on the fluid flow in a real cylindrical compression-dissolution unit where the liquid is sprayed from the top while simultaneously the gas is compressed from the bottom. Dynamic mesh technique is used to account for the compression with a moving piston.
\end{abstract}




\section{INTRODUCTION}

Increased amounts of worldwide $\mathrm{CO}_{2}$ emissions from different industries has drawn the attention of researchers to devise cycles where this gas could be used again for production of added value chemical products. In particular in Europe, a research and innovation challenge call (SPIRE-08-2017) was made on using carbon dioxide as a raw material to exploit its utilization for reduction of greenhouse gas emissions. The RECODE (Recycling carbon dioxide in the cement industry to produce added-value additives: a step towards a $\mathrm{CO} 2$ circular economy) project [1] is dedicated to recycling carbon dioxide from the cement industry to produce added value chemicals. In other words, the $\mathrm{CO}_{2}$ emissions coming from flue and process gases in the cement production plant is going to be reused within the plant itself to produce additives for production of cement-related products. Figure 1 shows the overall process scheme of this idea in the framework of the RECODE project. In the current study, the focus is on numerical simulations of a novel simultaneous compression-dissolution system, as pointed out in Fig. 1. A full compression cycle of this cylindrical unit consists of spraying the solvent from the top while at the same time compressing the gas from the bottom with the final aim of efficiently dissolving $\mathrm{CO}_{2}$ inside the liquid.

Volume of fluid method (VOF) [6], level-set [11] and front-tracking [13] methods are the common approaches for modeling and simulation of multiphase flows involving topology change in liquid structures. On the other hand, Lagrangian particle tracking (LPT) is used for systems involving both particle/droplets within the flow of a continuous fluid [3]. Multi-scale methods allow utilizing benefits of the two perspectives combined in different applications. Tomar et. al. [12] have studied the primary atomization of

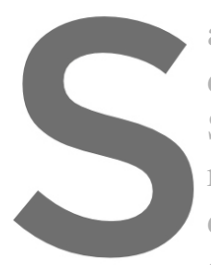
a turbulent liquid jet by density ratio of 100 . A Saeedipour et. al. [9] a ratio (water-air jets). described in Vallier's the algorithm with LPT is accomplished with its focus $\mathrm{cm}$
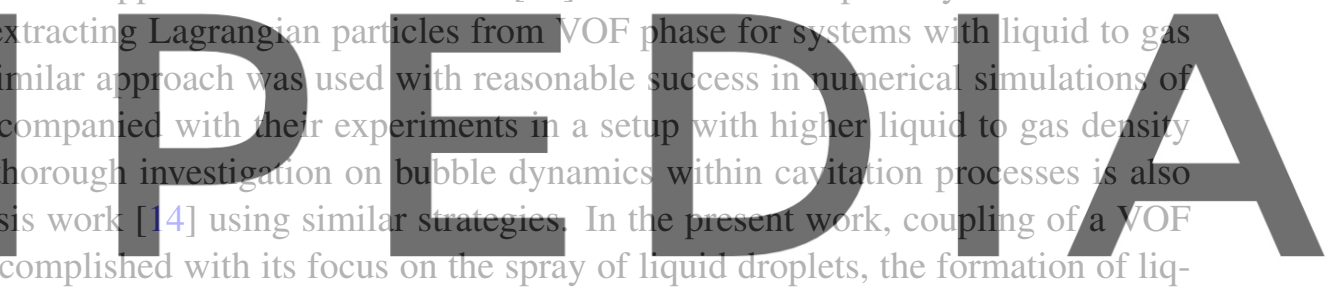
uid films on solid walls and its motion within a simultaneous compression-dissolution configuration as named interLPTFoam catching the spirit of interFoam which is OpenFOAM's original VOF solver and the LPT method.

\section{Methodology}

Typical Euler-Lagrangian solvers deal with multiphase flows consisting of a dispersed phase (usually spray of liquid) moving within the flow of a continuous phase (typically gas flows). Representation of the discrete phase and its motion is considered by Lagrangian particles and solution of Newton's second law of motion, respectively. The flow pattern of the continuous phase is described by solving the NavierStokes equations. This method is unable to deal with accumulation of droplets in a confined space and the creation of liquid films.

\subsection{Continuous phase}

A single field formulation approach of the VOF method is used to model the multiphase flow and compressible Navier-Stokes equations are solved for the continuous phase. Conservation equations of mass, 


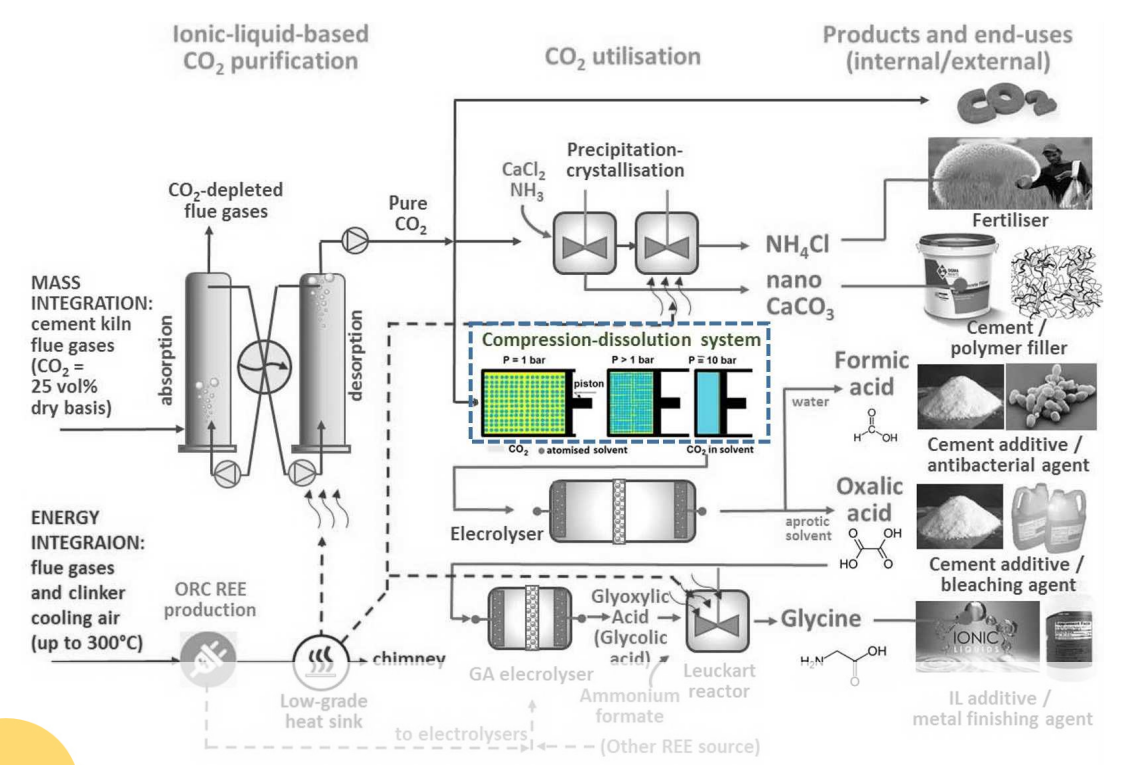

Figure 1: Overall process scheme of the RECODE [1]. Simultaneous compression-dissolution unit is shown in color within the dashed lines.

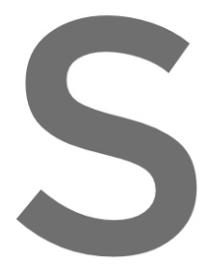

momentum and energy are
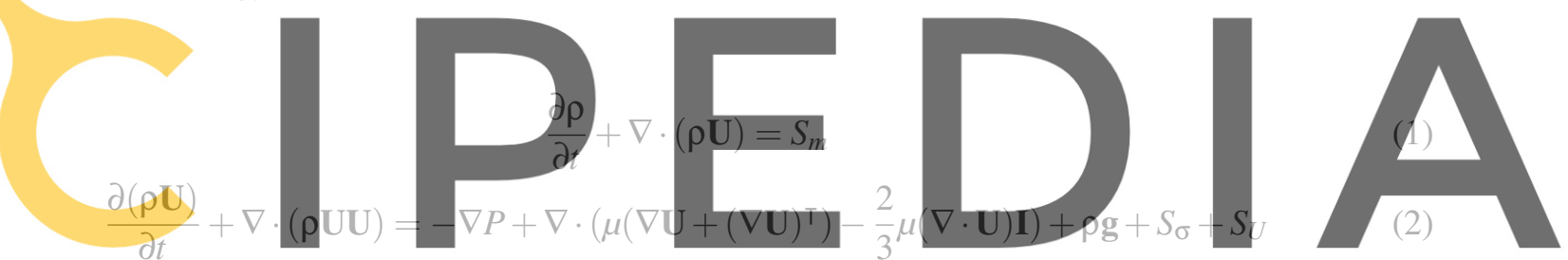

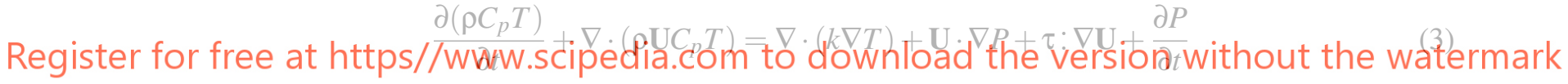

where $P$ is pressure and $\mathbb{U}$ is the velocity vector while $\rho, \mu, C_{p}$ and $k$ are density, dynamic viscosity, specific heat capacity and thermal conductivity, respectively. $\mathbf{g}$ is the gravitational acceleration and $\tau$ represents the viscous stress tensor. Source terms in the above-mentioned equations are formulated as following

$$
\begin{gathered}
S_{m}=\left(\rho_{l}-\rho_{g}\right) S_{\alpha} \\
S_{U}=\left(\rho_{l}-\rho_{g}\right) S_{\alpha} \mathbf{U}_{\mathbf{p}}+\frac{1}{V_{\text {cell }} \Delta t} \sum m_{p}\left(\left(\mathbf{U}_{\mathbf{p}}\right)_{t_{\text {out }}}-\left(\mathbf{U}_{\mathbf{p}}\right)_{t_{\text {in }}}\right) \\
S_{\sigma}=\sigma \cdot \kappa \cdot \nabla \alpha \quad \text { where } \quad \kappa=\nabla \cdot\left(\frac{\nabla \alpha}{|\nabla \alpha|}\right)
\end{gathered}
$$

where $S_{m}, S_{U}$ and $S_{\sigma}$ are the mass and momentum source terms due to conversion of Lagrangian particles to VOF and surface tension force[2], respectively. The momentum source term consists of the first term 

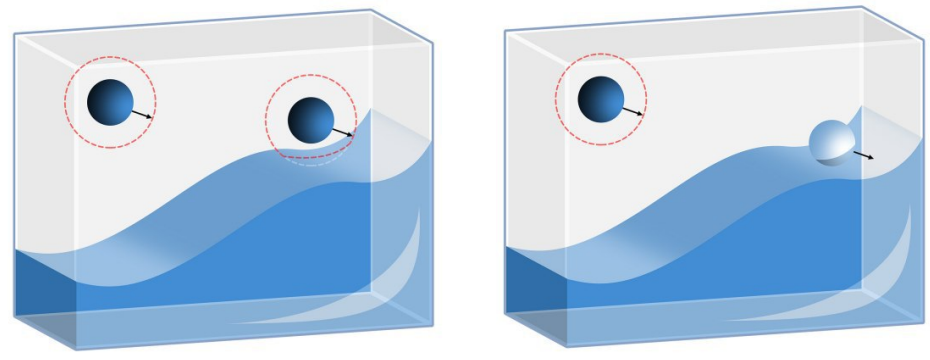

(a) LPtoVOF scenario

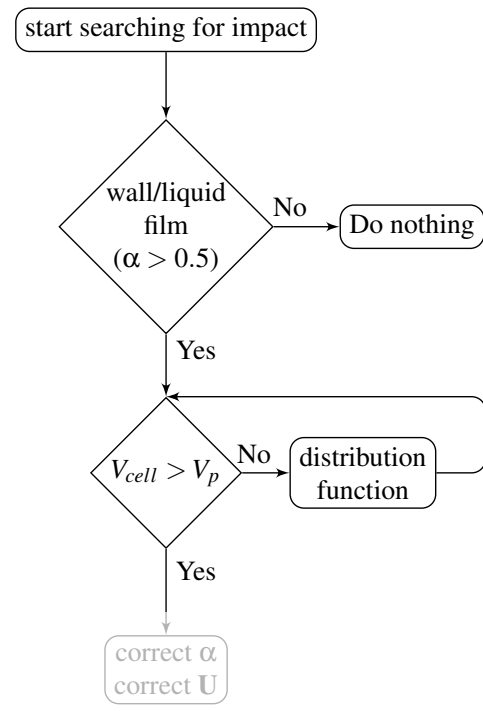

(b) LPtoVOF algorithm

Figure 2: Conversion of Lagrangian particles representing liquid droplets to the continuous multiphase field described by VOF: (a) Impact of a Lagrangian particle with a liquid film and its conversion to continuous liquid phase. Initial liquid film will be generated by impact of the first Lagrangian particles to the walls of the confined medium. Dark blue shows Lagrangian particles and light blue shows

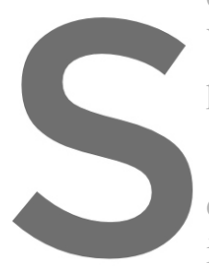
VOF-represented, droplet after conver putational timestep for

due to conversion and the second term showing the effect of discrete is worth noting that $(\mathbb{U}$
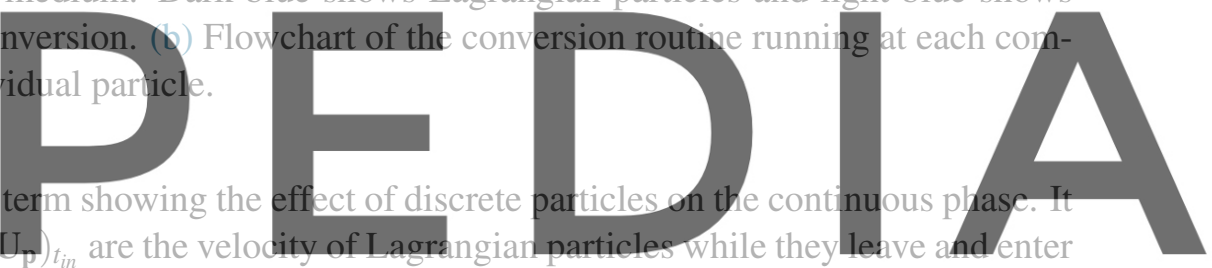

a computational cell. $\sigma$ and $\kappa$ are the surface tension coefficient and curvature, respectively. Two-way

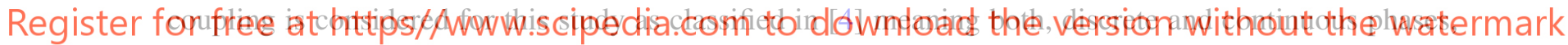
are influencing each other's motion. Motion of the interface between phases is being determined by solving the advection equation as described in eq. 7.

$$
\frac{\partial \alpha}{\partial t}+\nabla \cdot(\alpha \mathbf{U})+\nabla \cdot\left((1-\alpha) \alpha \mathbf{U}_{\mathbf{r}}\right)=S_{\alpha}
$$

In this equation, $\alpha$ is the liquid volume fraction in the mixture and $\mathbf{U}_{\mathbf{r}}$ is a suitable velocity field to compress the interface region. The last term on the left hand side of eq. 7 is an artificial compression term [8] which only acts on the computational cells containing the interface to achieve a sharper interface. The term on the right hand side of this equation shows the source term due to conversion of Lagrangian particles to VOF $\left(S_{\alpha}=\frac{V_{p}}{V_{\text {cell }} \Delta t}\right)$. CompressibleInterFoam [5] is used as the basic solver for solution of the above-mentioned conservation equations. This solver is designed for non-isothermal multiphase flows where the involving phases are assumed to be immiscible. 


\subsection{Discrete phase}

Lagrangian point-like particles are used to take into account the motion of sub-grid sprayed droplets. A collection of identical particles constructs a parcel which itself can be considered as a computational particle. Position and velocity of these parcels are calculated through solution of the Newton's second law of motion for each individual particle.

$$
\begin{gathered}
\frac{\mathrm{d} \mathbf{X}_{\mathbf{p}}}{\mathrm{d} t}=\mathbf{U}_{\mathbf{p}} \\
m_{p} \frac{\mathrm{d} \mathbf{U}_{\mathbf{p}}}{\mathrm{d} t}=\sum F
\end{gathered}
$$

In the equations above, $\mathbf{X}_{\mathbf{p}}, \mathbf{U}_{\mathbf{p}}$ and $m_{p}$ denote the position, velocity and mass of the Lagrangian particle. The right hand side term in eq. 9 shows the representative forces acting on the particles in a flow. Drag, gravity and buoyancy forces are considered in this study while other forces (added mass, pressure forces, etc.) are considered negligible for simplicity and brevity. In other words, eq. 9 can be expanded as

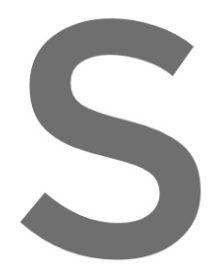

$$
m_{p} \frac{\mathrm{d} \mathbf{U}_{\mathbf{p}}}{\mathrm{d} t}=\frac{\pi d^{2}}{8} \rho C_{D}\left(\mathbf{U}-\mathbf{U}_{\mathbf{p}}\right)\left|\mathbf{U}-\mathbf{U}_{\mathbf{p}}\right|+\frac{\pi d^{3}}{6}\left(\rho_{p}-\rho_{g}\right) g
$$

\section{Register for free at https//www.scipedift.com to dob/inłolal}

In the above equation, $R \boldsymbol{e}_{p}=\frac{\rho\left(\left|\mathrm{U}-\mathrm{U}_{\mathrm{p}}\right|\right) D_{p}}{\mu}$ is the particie Reynoids number [10].

\subsection{Conversion of Lagrangian particles into VOF (LPtoVOF)}

Lagrangian particles are tracked within the fluid flow until either they hit the walls of the container or VOF-represented liquid films. Upon impact, their mass and momentum are transferred into VOF representation and the particles are going to be removed. A possible impact scenario of a Lagrangian droplet and a liquid film is sketched in Figure 2a. In the current approach, presence of solid walls or liquid films is checked at a certain distance from the particle depending on the size of particle and the cell size which the particle resides in. Transmission of mass and momentum takes place through an iterative process. At first, a spherical region of computational cells with its center lying at particle's position is selected. The volume of the liquid-free content of those cells are to be checked against the volume of the Lagrangian particle. If the mass content of the particle does not fit, the selected cells are filled with liquid $(\alpha=1)$ and the leftover mass is going to be checked within a spherical region with larger radius than the starting one. This iterative process happens until inner layers are filled with liquid 
$(\alpha=1)$ and only a small portion is distributed based on their distance to the center of the Lagrangian particle on the outer layer of the droplet. As a result, a sharp VOF-represented droplet at its core and a thin smeared outer layer will be produced. This process happens for each parcel and these steps could be followed as shown in Figure 2b.

\section{Validation}

Two preliminary validation test cases on ensuring mass and momentum conservation during a conversion of Lagrangian parcels into VOF are presented. At each instance of conversion, relative mass and momentum errors were calculated from their values before and after the impact. Introduced mass in VOF can easily be calculated by multiplication of the volume integration of $\alpha$ values multiplied by the density of the liquid. Hence, relative mass transfer error can be defined with respect to this value and mass of the Lagrangian parcel for each individual conversion. Likewise, the same approach can be taken for calculation of the momentum transfer error upon conversion.

\subsection{Mass conservation}

A specific volume $\left(6 \cdot 10^{-9} \mathrm{~m}^{3}\right)$ of Lagrangian parcels (water droplets) is sprayed within a $2 \mathrm{~cm} \times 2 \mathrm{~cm} \times$ $10 \mathrm{~cm}$ rectangular container with a pre-existing thin liquid film (see Figure $3 \mathrm{a}$ ) of $5 \mathrm{~mm}$. After that, enough time ( $8 \mathrm{~ms}$ ) is given to the sprayed parcels to get converted into the VOF $\alpha$-field either by hitting the walls of the container or the thin liquid pool resting at the bottom of the container. During this time period, mass content of the VOF phase is tracked in time to ensure that the final mass of Lagrangian
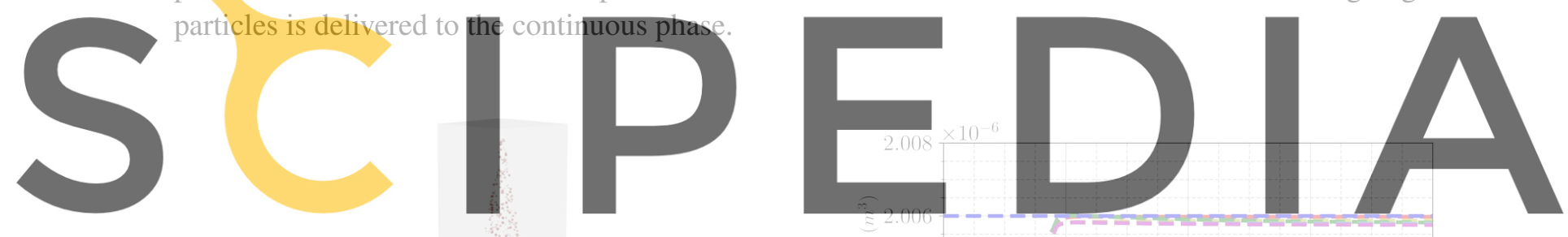

Register for free at https//www.scipedia.com to down

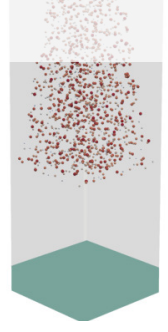

(a)

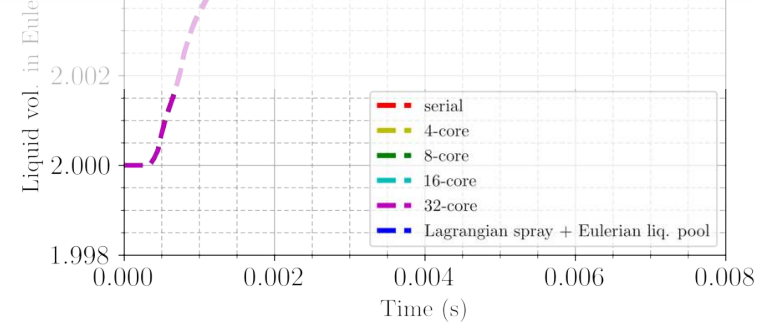

(b)

Figure 3: (a) Computational domain and (b) Liquid volume content of the computational domain while conversion of Lagrangian particles into VOF $\alpha$-field takes place.

Conversion of Lagrangian particles to VOF can get tricky when parcels are getting converted at places where the particles have to be transferred between domains associated with different computing processors, because the transfer of mass and momentum requires data from neighbouring processors. 
Table 1: Impact conditions for the liquid droplet impact onto hydrophobic solid surface [7].

\begin{tabular}{lccccccr}
\hline Material & $\theta_{a d v}$ & $\theta_{\text {rec }}$ & $\theta_{e q}$ & $D(\mathrm{~mm})$ & $V_{\text {impact }}(\mathrm{m} / \mathrm{s})$ & We & Outcome \\
\hline Wax & $105^{\circ}$ & $95^{\circ}$ & $100^{\circ}$ & 2.75 & 1.18 & 52 & partial rebound \\
\hline
\end{tabular}

Hence, simulations are performed in both serial and multiple processors in order to ensure a flawless conversion. Figure $3 \mathrm{~b}$ shows the results of simulations running on serial and multi-core configurations.

It can be seen from Fig. $3 \mathrm{~b}$ that in the beginning, $2 \cdot 10^{-6} \mathrm{~m}^{3}$ of water is present in VOF at the bottom of the domain. Then, injection begins, injecting a total of $6 \cdot 10^{-9} \mathrm{~m}^{3}$ of water inside the container. After $2 \mathrm{~ms}$, all of the Lagrangian parcels have been converted to the Eulerian phase, increasing the mass content to $2.006 \cdot 10^{-6} \mathrm{~m}^{3}$ in total.

\subsection{Momentum conservation}

As mentioned above, additionally to relative error in mass transfer upon conversion of LPs to VOF, the code was tested for a practical test case to ensure momentum conservation. One of the areas where the momentum transfer plays a key role is the impact of droplets on stationary solid surfaces [7]. In particular, the impact of a spherical water drop with $2.75 \mathrm{~mm}$ diameter, onto hydrophobic wax surface is chosen. The outcome of such an impact is the so-called partial rebound in which the droplet hits the surface, spreads over it, starts to recede and then a part of the droplet is detached from the surface while the other part is still attached. The conditions of this impact case are given in table 1.

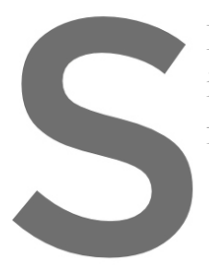
In order to accurately compute th
ical correlation of Kistler [15] for
following equations for calculation
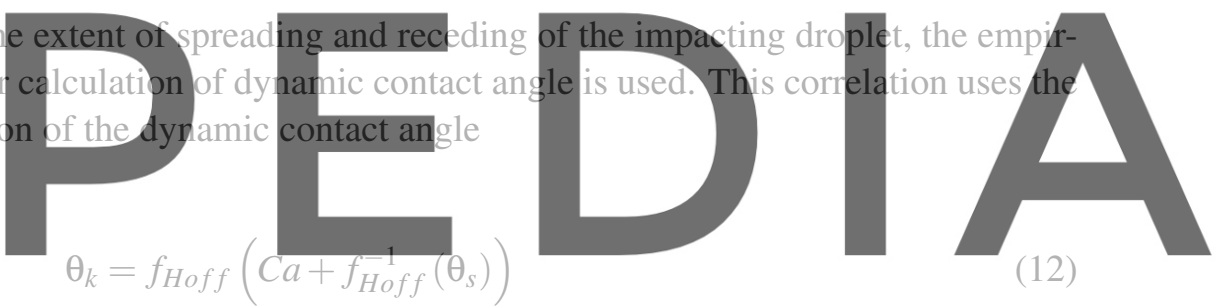

Register for free at https//www.scipedia.com to

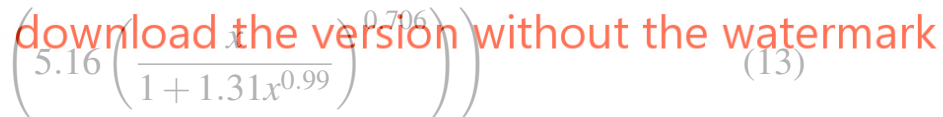

where $C a=V \mu / \sigma, \theta_{k}, \theta_{s}$ are the capillary number, dynamic kistler and static contact angles while $f_{H o f f}$ and $f_{\text {Hoff }}^{-1}$ are the Hoffman function (with $x$ being the independent variable in eq. 13) and the inverse function of the Hoffman function. Static contact angle is going to be substituted based on the sign of the capillary number and its value is either the static advancing or receding contact angle. The calculated dynamic contact angle at this stage is imposed as a boundary condition at the contact line which affects the curvature calculation (see eq. 6) for computation of surface tension force.

Two-dimensional axisymmetric simulations of this impact scenario is performed with the hybrid VOFLPT solver (interLPTFoam) within a $5^{\circ}$ wedge-shape geometry of size $1 \mathrm{~cm} \times 1 \mathrm{~cm}$. A uniform computational mesh $(350 \times 350)$ which results in almost 100 cells across the diameter of the droplet, is constructed to account for a sharp gas/liquid interface. For the sake of comparison, the same simulation case is run with the original VOF solver (interFoam) of OpenFOAM. Figure 4 shows reasonable agreement of the developed approach with respect to VOF simulation and experimental data by comparing the time evolution of the so-called spread factor. The spread factor $(s)$ is defined by the ratio of the 
spreading/contracting droplet diameter at specific instances of time to the initial diameter of the droplet. Likewise, non-dimensional time $(T)$ is derived with impact velocity and initial droplet diameter. It can be noted in Fig. 4 that the dynamics of the evolving contact line is computed with reasonable accuracy in both spreading $(0<T<2.5)$ and receding $(2.5<T<12.5)$ phases of droplet impact showing the potential of the current solver.
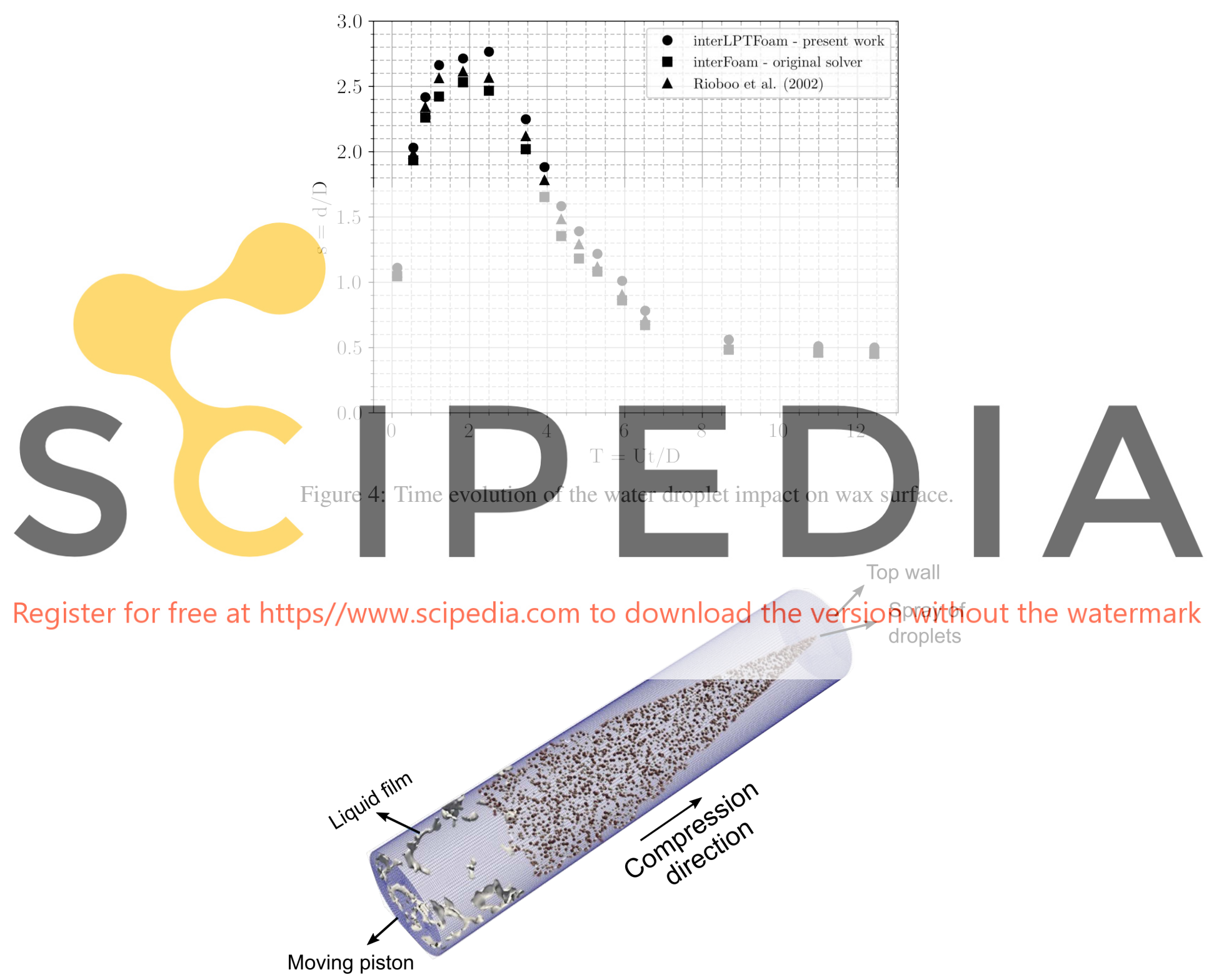

Figure 5: Qualitative view of the spray injection, computational grid and film creation over the walls in the simultaneous compression-dissolution unit 


\section{Results and discussion}

The newly developed interLPTFoam solver is used for hydrodynamic simulations of a simultaneous compression-dissolution unit. It consists of a cylindrical container with a nozzle at its top introducing the spray of liquid droplets, and a moving piston at its bottom, in order to simultaneously compress the gaseous phase during injection. Figure 5 shows a qualitative view of the spray injection, computational grid and film creation over the walls, where liquid film is represented by the iso-surface of $\alpha=0.5$. Dynamic mesh capability in OpenFOAM is employed to capture the domain modification in the compression phase. Table 2 describes the operating conditions for this configuration.

Table 2: Operating conditions in a simultaneous compression-dissolution cycle.

\begin{tabular}{lcccccr}
\hline Size $(\mathrm{cm})$ & $P_{\text {start }}(\mathrm{bar})$ & $P_{\text {end }}(\mathrm{bar})$ & Sprayed liquid (lit.) & $V_{\text {piston }}(\mathrm{m} / \mathrm{s})$ & $t_{\text {process }}(\mathrm{s})$ & $t_{\text {injection }}(\mathrm{s})$ \\
\hline$d=12.5, h=60$ & 1 & 10 & 0.8603 & 0.0643 & 8 & 7.5 \\
\hline
\end{tabular}

The size distribution of the initial liquid spray follows a Rosin-Rammler distribution function. This function is implemented as

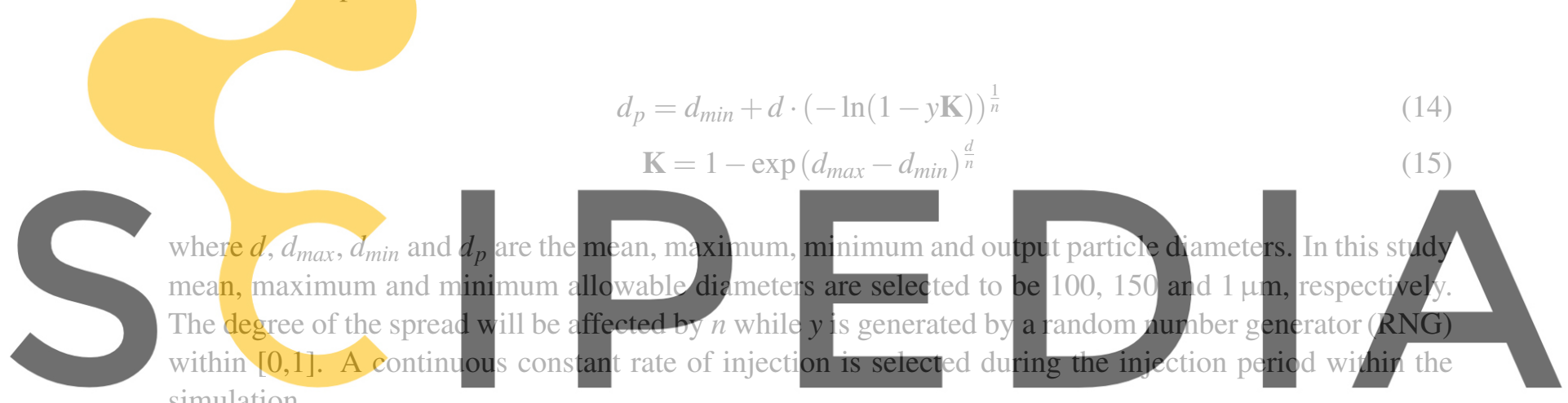
simulation.

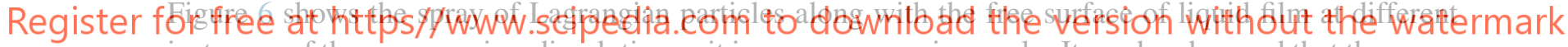

instances of the compression-dissolution unit in one compression cycle. It can be observed that the spray of Lagrangian parcels immediately forms liquid films over the walls of the cylinder leading to short

lifetime of the discrete droplets. Once this film starts to grow, it will disturb the spray of droplets by its agitating motion within the cylindrical container. And as the time passes, especially towards the end of the process these droplets instantly merge with the agitating liquid pool.

Active surface area between liquid and gas is a key factor in the dissolution process. This surface area can be calculated from the results of the simulation for Lagrangian particles and VOF-represented liquid film separately. Figure 7 shows the comparison of this surface area for discrete and continuous liquid structures. Although the sprayed droplets are creating huge surface areas, it can be seen that the liquid flow and presence of small bubbles could result even in higher active surface in favour of dissolution. 


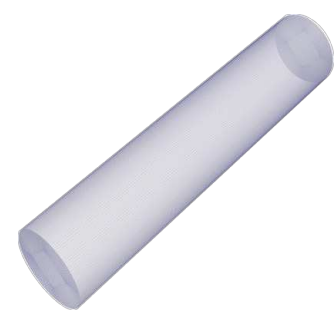

(a) $t=0$

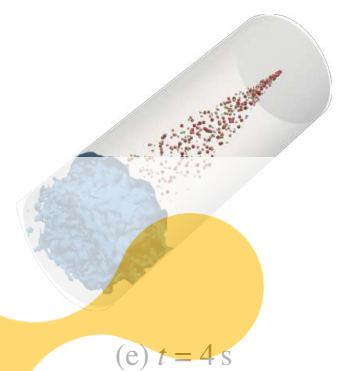

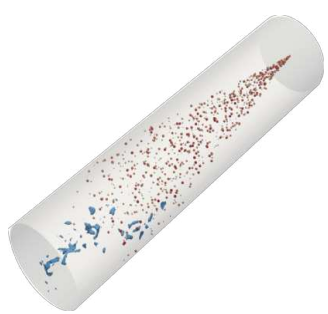

(b) $t=1 \mathrm{~s}$

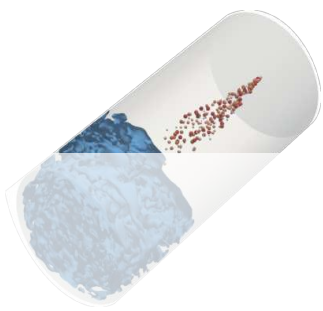

(f) $t=5 \mathrm{~s}$

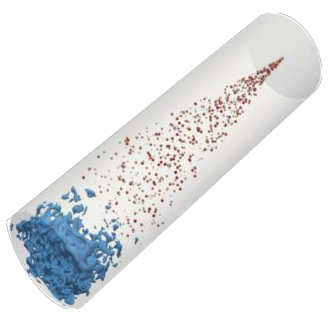

(c) $t=2 \mathrm{~s}$

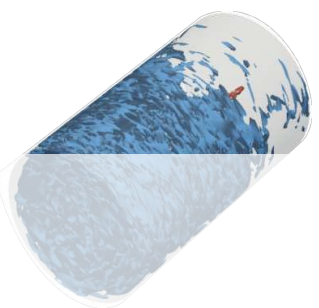

(g) $t=6 \mathrm{~s}$

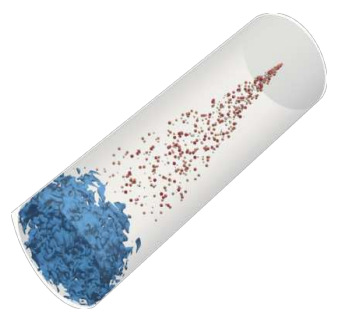

(d) $t=3 \mathrm{~s}$

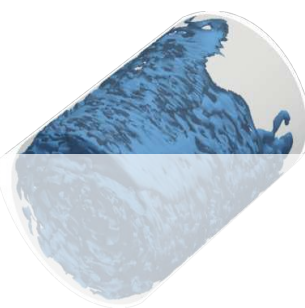

(h) $t=7 \mathrm{~s}$

Figure 6: Time evolution of the compression-dissolution unit
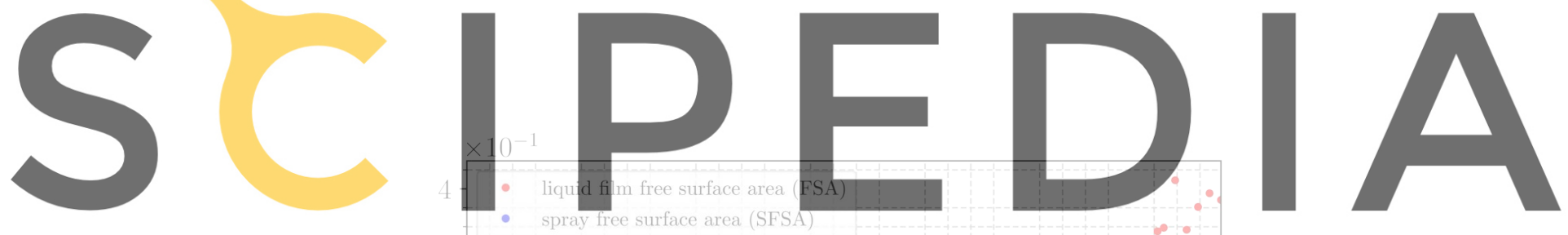

Register for free at https/Awww.scipedia.com to download the version without the watermark

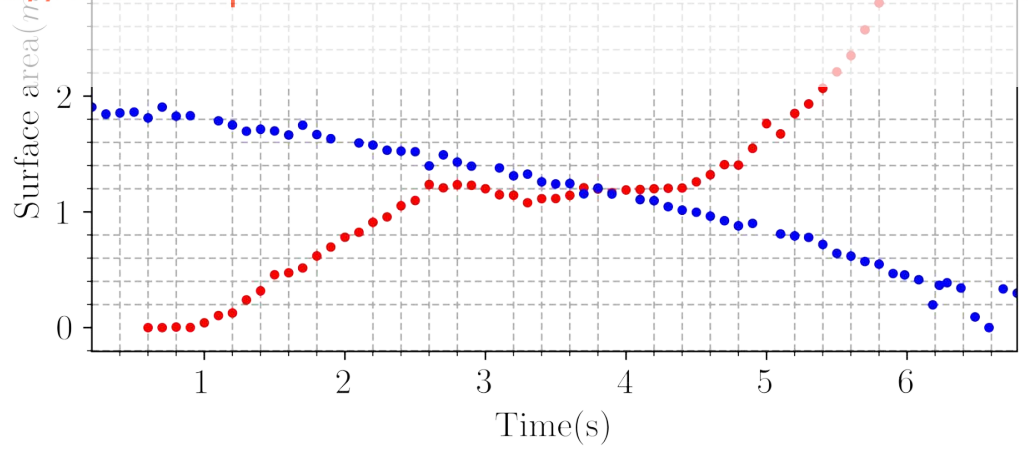

Figure 7: Time evolution of the free surface area between liquid and gaseous phases calculated for Lagrangian particles and VOF-represented liquid structures in one cycle of simultaneous compressiondissolution. 


\section{CONCLUSIONS}

A multiscale Eulerian-Lagrangian solver is developed within the framework of OpenFOAM, specifically for dense spray systems in confined spaces where film creation over the walls plays an important role. Spray of liquid droplets are represented by discrete Lagrangian particles while liquid films are expressed with the help of the liquid volume fraction in VOF. Several validation cases with positive results were performed to ensure mass and momentum conservation upon impact of Lagrangian parcels to the walls and liquid films. The method has been applied to CFD analysis of the hydrodynamics in a compressiondissolution unit to give insight on the internal multiphase flow. In particular, time evolution of the free surface area of the liquid film and spray of droplets are tracked. As a result it was observed that, not only these two surface areas are of the same order of magnitude showing the important role of the internal flow in enhancing the contact area between the liquid and gaseous phases but also that dynamics of the liquid film and the presence of small bubbles lead to even more enhanced surface between phases. This in turn emphasize the large influence of the dissolution process acting through the extended interface area between phases as a key for enhanced dissolution.

Moreover, it has been observed that the agitating liquid film continuously disturbs the incoming droplets. This effect is more pronounced towards the end of the compression cycle as the lifetime of the incoming droplets is too short since they are merging into the liquid film shortly after injection. This implies that the dissolution process would be more dominant by the surface area created from the fluid flow inside in addition to presence of small bubbles. In the future, we will add concentration equation for the continuous phase and assuming equilibrium condition for Lagrangian droplets to take into account dissolution of gas inside the liquid alongside the multiphase flow.

\section{Acknowledgement}

The project has received funding from the European Union's Horizon 2020 research and innovation program under grant agreement No 768583. The authors would gratefully acknowledge the financial support. Computational resources were provided by Steinbuch Centre for Computing (SCC) at Karlsruhe Institute of Technology (KIT).

\section{REFERENCES}

[1] Recycling carbon dioxide in the cement industry to produce added-value additives: a step towards a CO2 circular economy. https://recodeh2020.eu/.

[2] J.U. Brackbill, D.B. Kothe, and C. Zemach. A continuum method for modeling surface tension. Journal of Computational Physics, 100(2):335 - 354, 1992.

[3] C.T. Crowe, J.D. Schwarzkopf, M. Sommerfeld, and Y. Tsuji. Multiphase Flows with Droplets and Particles. CRC Press, 2nd edition, aug 2011.

[4] S. Elghobashi. On predicting particle-laden turbulent flows. Applied Scientific Research, 52(4):309-329, 1994.

[5] The OpenFOAM Foundation. OpenFOAM v7 User Guide, 2019.

[6] C.W. Hirt and B.D. Nichols. Volume of fluid (VOF) method for the dynamics of free boundaries. Journal of Computational Physics, 39(1):201 - 225, 1981. 
[7] R. Rioboo, M. Marengo, and C. Tropea. Time evolution of liquid drop impact onto solid, dry surfaces. Experiments in Fluids, 33(1):112-124, jul 2002.

[8] H. Rusche. Computational Fluid Dynamics of Dispersed Two-Phase Flows at High Phase Fractions. $\mathrm{PhD}$ thesis, Imperial College, London, 2002.

[9] M. Saeedipour, S. Schneiderbauer, G. Plohl, G. Brenn, and S. Pirker. Multiscale simulations and experiments on water jet atomization. International Journal of Multiphase Flow, 95:71 - 83, 2017.

[10] L. Schiller and A. Naumann. A drag coefficient correlation. Zeitschrift des Vereins Deutscher Ingenieure, 77:318-320, 1935.

[11] M. Sussman, P. Smereka, and S. Osher. A level set approach for computing solutions to incompressible two-phase flow. Journal of Computational Physics, 114(1):146 - 159, 1994.

[12] G. Tomar, D. Fuster, S. Zaleski, and S. Popinet. Multiscale simulations of primary atomization. Computers \& Fluids, 39(10):1864 - 1874, 2010.

[13] G. Tryggvason, B. Bunner, A. Esmaeeli, D. Juric, N. Al-Rawahi, W. Tauber, J. Han, S. Nas, and Y.J. Jan. A front-tracking method for the computations of multiphase flow. Journal of Computational Physics, 169(2):708 - 759, 2001.

[14] A. Vallier. Simulations of cavitation - from the large vapour structures to the small bubble dynamics. $\mathrm{PhD}$ thesis, Lund University, 2013.

[15] C. J. van Oss. A review of: “wettability " J.C. Berg, ed. Marcel Dekker. Journal of Dispersion Science and Technology, 14(6):717-718, 1993. 